\title{
Effects of the Coronavirus Disease-2019 Pandemic on Physical Activity Level, Depression, and Anxiety in Persons with Multiple Sclerosis
}

\author{
(D) Asiye Tuba Ozdogar1* , (D) Pınar Yigit1, (D) Zuhal Abasıyanık1,2, (D) Seda Dastan11, (D) Pelin Hancer1, (D) Ozge Sagıcı1 , (D) Cavid Baba3 , \\ (D) Serkan Ozakbas ${ }^{3}$, on behalf of Multiple Sclerosis Research Group \\ 1Dokuz Eylul University, Graduate School of Health Sciences, Izmir, Turkey \\ 2Izmir Katip Celebi University Faculty of Health Sciences, Department of Physiotherapy and Rehabilitation, Izmir, Turkey \\ ${ }^{3}$ Dokuz Eylul University Faculty of Medicine, Department of Neurology, Izmir, Turkey
}

\section{Abstract}

Objective: The mandatory restrictions during the Coronavirus disease-2019 (COVID-19) pandemic in regular physical activity and exercise affected the daily life of millions of people. Changes due to the COVID-19 outbreak in persons with multiple sclerosis (pwMS) are unknown. Therefore, this study aimed to examine the effects of the COVID-19 outbreak on physical activity level, depression, and anxiety in pwMS.

Materials and Methods: A total of 263 pwMS were assessed in the prospective follow-up study. Study participants in the last year were contacted by phone (from May 5 to June 5, 2020) depending on previous assessment dates, wherein 201 (154 female, 47 male) were reached during the pandemic. MS demographic and clinical characteristics were handled from the last routine examination in March 2019 to March 2020 . Physical activity, anxiety, and depression information before the COVID-19 pandemic were also obtained from the assessment within the same day.

Results: The physical activity assessed using the godin leisure-time exercise questionnaire (GLTEQ) improved during the pandemic ( $p=0.018$ ). Anxiety and depression levels significantly decreased during the pandemic ( $p<0.001)$. Significant correlations were found between the GLTEQ and anxiety and depression subscores in the hospital anxiety and depression scale $(r=-0.149, r=-0.161, p<0.05$, respectively) during the pandemic. However, a correlation was not found between these variables before the pandemic $(p>0.05)$.

Conclusion: This study provides some evidence about the pandemic effects on physical activity level, depression, and anxiety in pwMS. The precaution taken to control the pandemic was already covered for three months; however, anxiety, depression, and inactivity behavior, which were also prevalent before COVID-19, was reduced.

Keywords: Multiple sclerosis, COVID-19, pandemic, physical activity, anxiety, depression

\section{Introduction}

The Coronavirus disease-2019 (COVID-19) outbreak is the most severe pneumonia outbreak to date. At the end of 2019, a new coronavirus, known as severe acute respiratory syndrome coronavirus 2, suddenly appeared in Wuhan, China. The World Health Organization declared an internationally concerned public health emergency on January 31, 2020. As of April 16, 2020, COVID-19 spread worldwide and caused $>2$ million cases and $>137,000$ deaths (1). In Turkey, the first case was confirmed on March 9, 2020, and the first death on March 17, 2020 (2).

Wang et al. (3) evaluated 1,210 participants for the effect of the COVID-19 pandemic on their psychological situation, which revealed that $53.8 \%$ of participants had moderate to severe psychological effects during the pandemic. Another study revealed that anxiety and stress disorder rates of healthcare professionals of a hospital in China were $23 \%$ and

Address for Correspondence: Asiye Tuba Ozdogar, Dokuz Eylul University, Graduate School of Health Sciences, Izmir, Turkey E-mail: asiye.tuba.ozdogar@gmail.com ORCID-ID: orcid.org/0000-0003-0043-9374

Received: 04.08.2021 Accepted: 04.10.2021

${ }^{\circ}$ Copyright 2021 by the Journal of Multiple Sclerosis Research published by Galenos Publishing House. 
$27 \%$, respectively (4). The neuropsychiatric effects of the viral pandemic were previously shown on the general population; however, its effects on persons with multiple sclerosis (pwMS) are unclear.

Neuropsychiatric involvement is one of the most common symptoms in pwMS. Anxiety and depression were reported in $57 \%$ and $40 \%$ of patients, respectively. These are above the rates according to the general population $(5,6)$. Therefore, they are more vulnerable to the neuropsychiatric effects of the COVID-19 pandemic. Patel et al. (7) showed that depression and anxiety levels affect the quality of life, fatigue level and disability scores, and disease progression. Just like other society members, pwMS are affected by the emotional distress and health anxiety caused by the COVID-19 outbreak. However, pwMS follow incompatible coping strategies that made them more sensitive to the harmful neuropsychiatric effects of the outbreak (8). Most pwMS receive immunosuppressive or immunomodulatory therapy. Patients taking immunosuppressive agents theoretically have an increased risk of being affected by viral pandemics, with a higher health anxiety level. Moreover, pwMS have difficulty accessing group and cognitive and physical rehabilitation therapies that hypothetically contribute to stress and anxiety (9).

Physical activity is defined as any physical movement resulting from skeletal muscle contraction, including exercise, sports, professional work, transportation, and housework, which results in increased energy consumption above the resting levels (>1.6 MET) (10). Physical activity is highly recommended in pwMS. Evidence shows that physical activity improves walking performance, balance, cognitive state, fatigue, depression, and quality of life in pwMS (11). However, low physical activity and exercise levels and high levels of sedentary behavior were reportedly common in pwMS (12).

Public health officials recommended reducing travel and stay at home as a primary means of limiting human exposure to the virus due to the ongoing COVID-19 outbreak. Mandatory restrictions in regular physical activity and exercise participation affected the daily life of millions of people. Changes due to COVID-19 outbreak in pwMS, whose anxiety and depression level is higher than the general population, but with low physical activity and exercise levels, are unknown. Therefore, this study aimed to examine the effects of the COVID-19 outbreak on physical activity level, depression, and anxiety in pwMS.

\section{Materials and Methods}

\section{Participants and Procedures}

This study was implemented in the MS Center of Dokuz Eylul University Hospital, Izmir, Turkey. The research protocol was approved by the Turkish Ministry of Health and Dokuz Eylul University Ethics Committee (code: 2020/15-32). Written consent was obtained from all participants for data before the pandemic and verbal consent before the evaluation during the pandemic.

This study used the physical activity, anxiety, and depression data of the ongoing project entitled, "Follow-up of physical, psychosocial, and cognitive influences in persons with multiple sclerosis: A prospective cohort study," as baseline data. This project conducted physical, cognitive, and psychosocial assessments by physiotherapists, psychologists, MS nurses at 6-month intervals, in addition to routine clinical examination by a senior MS neurologist. Moreover, the questionnaires were filled by the patients. Study participants in the last year were contacted by phone (from May 5 to June 5, 2020) depending on previous assessment dates. The starting day was approximately 8 weeks after the date the Government of Turkey decided to administratively allow those with chronic illnesses and close the schools (March 16, 2020). While many government agency employees did not go to work, private-sector workers or health personnel continued to work at limited hours, except for curfew days. In Turkey, curfew was implemented for 8 days in April and 15 days in May.

The eligibility criteria were as follows: definite MS diagnosis according to the recent diagnostic criteria (13) and age between 18 and 70 years. Exclusion criteria included the following: Having a relapse within 30 days, an additional diagnosis of neurological disease other than MS, having any severe cognitive disorder hindering the test instructions, and communication problems.

\section{Outcome Easures}

The MS demographic and clinical characteristics, including gender, age, marital and employment status, disease duration, disease course, and expanded disability status scale (EDSS), were handled from the last routine examination from March 2019 to March 2020. Physical activity, anxiety, and depression information before the COVID-19 pandemic were also obtained from the assessment within the same day.

\section{Physical Activity Assessment}

Godin leisure-time exercise questionnaire (GLTEQ) was applied for physical activity evaluation. GLTEQ is a very common and validated questionnaire in MS researches $(13,14)$, which includes three items regarding the number of bouts of strenuous, moderate, and mild physical activity lasting for $>15$ min in a typical week. The total score is calculated using the following formula: (frequency of mild $\times 3$ ) + (frequency of moderate $\times 5)+($ frequency of strenuous $\times 9$ ). Scores of 24 and above mean sufficiently active, 23-14 is moderately active, and 13 and below are insufficiently active (15).

\section{Anxiety and Depression}

Anxiety and depression were evaluated using the Turkish version of the hospital anxiety and depression scale (HADS) (16), which is a reliable and validated self-reported scale in MS 
(17) that includes 14 items, 7 of which evaluate anxiety and 7 assess depression. Both subscales are separately calculated by summating the 7 items, and higher scores indicate more significant anxiety and depression level. Eight points or above for subscales indicate clinically significant anxiety and depression in MS (17).

\section{Statistical Analysis}

International Business Machines Corporation ${ }^{\circledast}$ Statistical Package for the Social Science ${ }^{\circledast}$ Statistics for Windows (Version 25.0. Armonk, NY: IBM Corp.) was used for statistical analysis. The normality distribution of the data was checked with the ShapiroWilk test, investigating the histograms and plots. Descriptive analysis (mean, median, standard deviation, range, percentages, and numbers) was performed for each baseline characteristic, including sex, age, disease course, disease duration, and EDSS score. The Wilcoxon signed-rank test was used to compare the scores before and during the COVID-19 pandemic, and effect sizes were calculated using Cohen's d. Effect sizes of 0.2, 0.5, and 0.8 describe small, medium, and large effects, respectively (18). Bivariate correlation analysis was performed to investigate the relationship between physical activity and depression and anxiety using the Spearman rank-order correlation. The level of significance was set as $p \leq 0.05$.

\section{Results}

By March 2020, 263 pwMS were assessed in the prospective follow-up study, wherein 201 (154 female, 47 male) were reached by phone during the pandemic. Sixty-two patients were excluded from the study, either because they were unreachable by phone $(n=55)$ or they did not want to answer the questions on the phone $(n=7)$. The mean EDSS of the study participants was 1.09 \pm 1.34 (range between 0 and 7) (Table 1). None of them was positive for COVID-19.

Before and during the pandemic values were analyzed, which

\begin{tabular}{|l|l|}
\hline $\begin{array}{l}|l| \\
\text { Table 1. Demographic and clinical characteristics of } \\
\text { participants }\end{array}$ & Mean (SD) \\
\hline Age (years) & $35.54 \pm 10.12$ \\
\hline Gender, $n(\%)$ & $154(76.6 \%)$ \\
\hline Female & $47(23.4 \%)$ \\
\hline Male & \\
\hline Disease course, $n(\%)$ & $198(98.5 \%)$ \\
\hline Relapsing-remitting MS & $2(1 \%)$ \\
\hline Secondary progressive MS & $1(0.5 \%)$ \\
\hline Primary progressive MS & $1.09 \pm 1.34$ \\
\hline EDSS & $7.08 \pm 7.13$ \\
\hline Disease duration (years) &
\end{tabular}

EDSS: Expanded disability status scale, MS: Multiple sclerosis, SD: Standard deviation revealed that the physical activity assessed by GLTEQ improved during the pandemic $(p=0.018)$. Anxiety and depression levels statistically significantly decreased during the pandemic $(p<0.001)$ (Table 2).

Assessments before the pandemic revealed 158 insufficiently active pwMS, whereas 142 pwMS were inactive during the pandemic. Forty-two pwMS, without regular exercise habits before the pandemic, started to exercise regularly during the pandemic. Twenty participants who regularly exercise before the pandemic, stopped exercising during the pandemic (Figure 1). Clinically significant depression before the pandemic according to the 8-point threshold of HADS was observed in 58 pwMS; however, this number dropped to 36 during the pandemic. The number of patients with anxiety decreased from 84 to 48 (Figure 2).

As shown in Table 3, significant correlations were found between the GLTEQ and anxiety and depression subscores of HADS $(r=-0.149,-0.161, p<0.05$; respectively) during the pandemic. However, the correlation between these variables before the pandemic was statistically non-significant ( $p>0.05$ ).

\section{Discussion}

This study compared physical activity, anxiety, and depression levels of pwMS before and during the pandemic. Results revealed anxiety in $23.88 \%$ of participants, whereas $17.91 \%$ had depression during the pandemic. In addition, 29.35\% of participants were active during the pandemic.

Recent systematic reviews and meta-analyses reported that the rates of anxiety and depression in pwMS were 31\% and $22 \%$, respectively (19). Furthermore, Chiaravalloti et al. (20) assessed 131 pwMS regarding anxiety and depression levels and compared the data collected before the pandemic. They reported no changes in terms of anxiety level and a slightly increased depression level during the pandemic according to the pre-pandemic period. Our study revealed an anxiety rate of $42 \%$ and depression of $29 \%$ in the pre-pandemic period. These rates significantly decreased after the COVID-19 pandemic, which depended on two factors. First, immediately after the beginning of the outbreak, a comprehensive information letter was published about the potential effects of the COVID-19 pandemic on pwMS. The letter contained detailed information made by the MS clinic of Dokuz Eylul University, where patients were followed up, which was spread to a broad audience by the MS Research Association, with close cooperation via its web page and social media pages. The information letter published by the center, where the people are followed, effectively reduced the increased anxiety and depression due to incorrect information circulating in the society. Second, the increased physical activity levels positively affected the psychological state. Costabile et al. (21) showed that exercise plays a crucial role during the pandemic to overcome mental distress, improve active lifestyle, 
Table 2. Results of physical activity and depression-anxiety before and after COVID-19 pandemic

\begin{tabular}{|l|l|l|l|l|}
\hline & Pre-pandemic & During pandemic & $p$ & Cohen's d \\
\hline GLTEQ & $0(0-9)$ & $0(0-15)$ & $0.018^{*}$ & 1.26 \\
\hline HADS-anxiety & $7(4-10)$ & $3(1-7)$ & $<0.001^{*}$ & 0.83 \\
\hline HADS-depression & $4(2-8)$ & $2(1-5)$ & $<0.001^{*}$ & 0.32 \\
\hline
\end{tabular}

Wilcoxon signed-rank test, Values are presented as median (Q1-Q3), *Statistically significant ( $p \leq 0.05)$, GLTEQ: Godin leisure-time exercise questionnaire, HADS: Hospital anxiety and depression scale, COVID-19: Coronavirus disease-2019

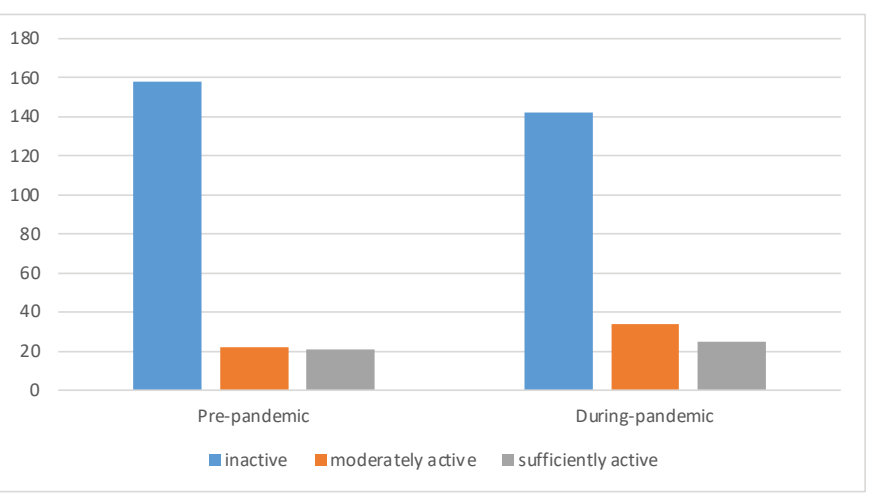

Figure 1. Physical activity rates of participants before and during the pandemic

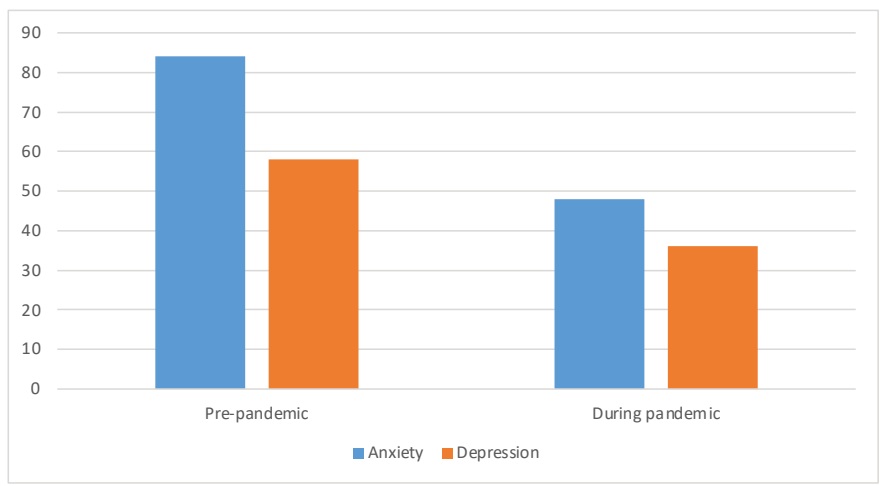

Figure 2. Depression and anxiety rates before and during the pandemic

increase positive emotions, and decrease negative feelings, such as depression and anxiety. More recently, a systematic review investigating the effects of exercise on depression and anxiety showed a significantly negative correlation of changes from moderate to large (22). Furthermore, Kandola et al. (23) provided extensive information about the relation between physical activity and psychological state. In addition, they point out information gaps concerning intensity and pathophysiological mechanisms of physical activity. Similarly, a weak negative relationship was found between depression and anxiety and physical activity levels during the pandemic in our study. However, a relationship was not found between physical activity and anxiety and depression before the pandemic since the psychological state of people is affected by more factors related to daily life before the pandemic. Contrarily, health-related factors affected the psychological state more during the pandemic. In addition, the intensity of physical activity is not enough to reduce anxiety and depression levels before the pandemic.

The effects of physical activity on fatigue, strength, balance, quality of life, and mobility were shown in pwMS; however, they have less physical activity level versus the general population (24). The present study revealed that $71.14 \%$ of participants were inactive in the pre-pandemic period, whereas $70.65 \%$ were inactive during the pandemic. However, the physical activity level in pwMS increased according to GLTEQ during the pandemic. While 42 patients started to exercise, 20 patients gave up exercise habits during the pandemic. Despite the precaution and physical restrictions, some of the pwMS continued their physical activities by changing the form of activity due to the permanent acquisition of physical activity behavior.

\section{Study Limitations}

Our study has several limitations. First, the type of exercise was not reported. Patients reported that indoor activities replaced outdoor activities due to quarantine restrictions. The physical activity levels were not objectively measured due to pandemic conditions. In addition to the physical activity level, sitting time could also be questioned. However, it was not included because a comparison could not be made since our previous evaluations did not include a sitting time assessment. Lastly, life situation-related questions, such as working status, were not included. The strength of this study is the awareness of the anxiety, depression, and physical activity levels of our patients just before the pandemic, which enables us to see the effects of the pandemic on the same patients.

Table 3. Spearman's correlation coefficients between the GLTEQ and HADS-anxiety and depression

\begin{tabular}{|c|c|c|c|c|}
\hline & \multicolumn{2}{|l|}{ Pre-pandemic } & \multicolumn{2}{|c|}{ During pandemic } \\
\hline & HADS-anxiety & HADS-depression & HADS-anxiety & HADS-depression \\
\hline GLTEQ & $\begin{array}{l}r=-0.037 \\
p=0.6\end{array}$ & $\begin{array}{l}r=-0.064 \\
p=0.365\end{array}$ & $\begin{array}{l}r=-0.149 * \\
p=0.035\end{array}$ & $\begin{array}{l}r=-0.161^{*} \\
p=0.023\end{array}$ \\
\hline
\end{tabular}

Spearman rank-order correlation, *Statistically significant $(p \leq 0.05)$, GLTEQ: Godin leisure-time exercise questionnaire, HADS: Hospital anxiety and depression scale 
This study provides some evidence about the effect of the pandemic on anxiety, depression, and physical activity levels in pwMS. The precaution taken to control the pandemic was already covered for three months; however, anxiety, depression, and inactivity behavior, which were also prevalent before COVID-19, was reduced. This study also includes hints that early and explicit information during extraordinary periods has a preventive effect on reactive anxiety and depression in pwMS.

Acknowledgments: The authors acknowledge the Multiple Sclerosis Research Association for assistance during the recruitment of the study.

\section{Ethics}

Ethics Committee Approval: The research protocol was approved by Dokuz Eylul University Ethics Committee (code: 2020/15-32). This study was performed in line with the principles of the Declaration of Helsinki (as revised in Brazil 2013).

Informed Consent: Written consent was obtained from all participants for data before the pandemic and verbal consent before the evaluation during the pandemic.

\section{Authorship Contributions}

Surgical and Medical Practices: C.B., S.O., Concept: A.T.O., Z.A., C.B., S.O., Design: A.T.O., P.Y., Z.A., S.D., P.H., O.S., C.B., S.O., Data Collection or Processing: A.T.O., P.Y., Z.A., S.D., P.H., O.S., C.B., Analysis or Interpretation: A.T.O., Z.A., Literature Search: A.T.O., P.Y., Z.A., S.D., P.H., O.S., C.B., S.O., Writing: A.T.O., Z.A., S.O.

Conflict of Interest: No conflict of interest was declared by the authors.

Financial Disclosure: The authors declared that this study received no financial support.

\section{References}

1. Rothan HA, Byrareddy SN. The epidemiology and pathogenesis of coronavirus disease (COVID-19) outbreak. Journal of autoimmunity 2020;109:102433.

2. Demirbilek Y, Pehlivantürk G. COVID-19 outbreak control, example of ministry of health of Turkey 2020;50:489-494.

3. Wang C, Pan R, Wan X, Tan Y, Xu L, Ho CS. Immediate psychological responses and associated factors during the initial stage of the 2019 coronavirus disease (COVID-19) epidemic among the general population in China. Int J Environ Res Public Health 2020;17:1729.

4. Lai J, Ma S, Wang Y, Cai Z, Hu J, Wei N, Wu J, Du H, Chen T, Li R, Tan H, Kang L, Yao L, Huang M, Wang H, Wang G, Liu Z, Hu S. Factors associated with mental health outcomes among health care workers exposed to coronavirus disease 2019. JAMA Netw Open 2020;3:e203976. doi: 10.1001/ jamanetworkopen.2020.3976.

5. Feinstein A, Magalhaes S, Richard JF, Audet B, Moore C. The link between multiple sclerosis and depression. Nat Rev Neurol 2014;10:507-517.

6. Butler E, Matcham F, Chalder T. A systematic review of anxiety amongst people with Multiple Sclerosis. Mult Scler Relat Disord 2016;10:145-168.

7. Patel VP, Walker LA, Feinstein A. Revisiting cognitive reserve and cognition in multiple sclerosis: A closer look at depression. Mult Scler 2018;24:186195.
8. Amato MP, Prestipino E, Bellinvia A. Identifying risk factors for cognitive issues in multiple sclerosis. Expert Rev Neurother 2019;19:333-347.

9. Haji Akhoundi F, Sahraian MA, Naser Moghadasi A. Neuropsychiatric and cognitive effects of the COVID-19 outbreak on multiple sclerosis patients. Mult Scler Relat Disord 2020;41:102164.

10. Caspersen CJ, Powell KE, Christenson GM. Physical activity, exercise, and physical fitness: definitions and distinctions for health-related research. Public Health Rep 1985;100:126-131.

11. Motl RW, Sandroff BM. Benefits of exercise training in multiple sclerosis. Curr Neurol Neurosci Rep 2015;15:62.

12. Hubbard EA, Motl RW, Manns PJ. The descriptive epidemiology of daily sitting time as a sedentary behavior in multiple sclerosis. Disabil Health J 2015;8:594-601.

13. Gosney JL, Scott JA, Snook EM, Motl RW. Physical activity and multiple sclerosis: validity of self-report and objective measures. Fam Community Health 2007;30:144-150.

14. Sikes EM, Richardson EV, Cederberg KJ, Sasaki JE, Sandroff BM, Motl RW. Use of the Godin leisure-time exercise questionnaire in multiple sclerosis research: a comprehensive narrative review. Disabil Rehabil 2019;41:12431267.

15. Amireault S, Godin G. The Godin-Shephard leisure-time physical activity questionnaire: validity evidence supporting its use for classifying healthy adults into active and insufficiently active categories. Percept Mot Skills 2015;120:604-622.

16. Paker N, Bugdayci D, Midik M, Celik B, Kesiktas N. Reliability of the Turkish version of the hospital anxiety and depression scale in the people with traumatic spinal cord injury. NeuroRehabilitation 2013;33:337-341.

17. Honarmand K, Feinstein A. Validation of the Hospital Anxiety and Depression Scale for use with multiple sclerosis patients. Mult Scler 2009;15:1518-1524.

18. Cohen J. Statistical power analysis for the behavioral sciences. Massachusetts, Academic Press, 2013.

19. Boeschoten RE, Braamse AMJ, Beekman ATF, Cuijpers P, van Oppen P, Dekker J, Uitdehaag BMJ. Prevalence of depression and anxiety in multiple sclerosis: a systematic review and meta-analysis. J Neurol Sci 2017;372:331341.

20. Chiaravalloti ND, Amato MP, Brichetto G, Chataway J, Dalgas U, DeLuca J, Meza C, Moore NB, Feys P, Filippi M, Freeman J, Inglese M, Motl R, Rocca MA, Sandroff BM, Salter A, Cutter G, Feinstein A; CogEx Research Team. The emotional impact of the COVID-19 pandemic on individuals with progressive multiple sclerosis. J Neurol 2021;268:1598-1607.

21. Costabile $T$, Carotenuto A, Lavorgna L, Borriello G, Moiola L, Inglese M, Petruzzo M, Trojsi F, lanniello A, Nozzolillo A, Cellerino M, Boffa G, Rosa L, Servillo G, Moccia M, Bonavita S, Filippi M, Lanzillo R, Brescia Morra V, Petracca M. COVID-19 pandemic and mental distress in multiple sclerosis: Implications for clinical management. Eur J Neurol 2021;28:3375-3383.

22. Wegner $M$, Helmich I, Machado $S$, Nardi $A E$, Arias-Carrion $O$, Budde $H$. Effects of exercise on anxiety and depression disorders: review of metaanalyses and neurobiological mechanisms. CNS Neurol Disord Drug Targets 2014;13:1002-1014.

23. Kandola A, Vancampfort D, Herring M, Rebar A, Hallgren M, Firth J, Stubbs B. Moving to beat anxiety: epidemiology and therapeutic issues with physical activity for anxiety. Curr Psychiatry Rep 2018;20:63.

24. Casey B, Coote S, Galvin R, Donnelly A. Objective physical activity levels in people with multiple sclerosis: meta-analysis. Scand J Med Sci Sports 2018;28:1960-1969. 